\title{
Políticas públicas de seguridad en Medellín: lecturas del problema de in-seguridad desde el enfoque de la seguridad humana
}

\author{
Natalia Andrea Cardona Berrio* \\ (natalia.crdn@gmail.com)
}

\author{
Carolina Sánchez Henao** \\ (carosan-21@hotmail.com)
}

Articulo de reflexion recibido el 27/11/2014 y aprobado el 19/12/2014

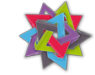

Cómo citar este artículo:

CARDONA BERRIO, Natalia Andrea y Carolina Sánchez Henao (2014). "Políticas públicas de seguridad en Medellín: lecturas del problema de in-seguridad desde el enfoque de la seguridad humana". En: Trans-pasando Fronteras, núm. 6, pp. 119138. Cali, Colombia: Centro de Estudios Interdisciplinarios, Jurídicos, Sociales y Humanistas (CIES), Facultad de Derecho y Ciencias sociales, Universidad Icesi.
\end{abstract}

\begin{abstract}
Resumen
En el artículo, se presenta la propuesta interpretativa y los principales hallazgos de la investigación: Análisis de las políticas públicas de seguridad implementadas por la administración municipal de Medellín, 2012-2013. Se empieza ubicando la propuesta de análisis desde la seguridad humana desde abajo, que permite destacar las interpretaciones que frente al problema público de la inseguridad en Medellín tienen sus comunidades y sus percepciones acerca de las políticas que el gobierno local implementa en esta materia. Posteriormente, se presentan los hallazgos más importantes de la investigación desde tres ideas centrales: el sesgo conceptual por parte de la administración municipal frente al concepto de la seguri-
\end{abstract} gación Social. Investigadora del Observatorio de Seguridad Humana de Medellín y de la Personería de Medellín.

** Politóloga, Universidad de Antioquia. Investigadora del Observatorio de Seguridad Humana de Medellín y de la Personería de Medellín. 
dad humana, la cual expone un discurso transversalizado por este enfoque y en la práctica transita del enfoque humano al enfoque restringido de la seguridad; la divergencia en la definición del problema de la inseguridad en la ciudad de Medellín por parte de las comunidades y la administración municipal; y las consecuencias de asumir un enfoque restringido para comprender y actuar frente al problema de seguridad. Finalmente, a partir de los hallazgos realizados, se presentan unas recomendaciones generales que permitan intervenir de manera más acertada los problemas de inseguridad en la ciudad.

\section{Palabras clave:}

Políticas públicas, Seguridad, seguridad humana, problema de inseguridad.

\section{Introducción}

La presente ponencia contiene la propuesta interpretativa y los principales hallazgos de la investigación Análisis de las políticas públicas de seguridad implementadas por la administración municipal de Medellín, 2012-2013. En primer lugar, se presenta la propuesta de análisis desde la seguridad humana desde abajo, que permite destacar las interpretaciones que frente al problema público de la seguridad en Medellín tienen sus comunidades y sus percepciones acerca de las políticas que el gobierno local implementa en esta materia. En segundo lugar, se presentan los hallazgos más importantes de la investigación desde tres ideas centrales: primero, el sesgo conceptual por parte de la administración municipal frente al concepto de la seguridad humana, toda vez que expone un discurso transversalizado por este enfoque y en la práctica se da un tránsito del enfoque humano al enfoque restringido de la seguridad, que propende por salidas represivas y militaristas, centradas en el aumento del pie de fuerza y la dotación técnica y tecnológica de la policía. Segundo, la divergencia en la definición del problema de la seguridad en la ciudad de Medellín por parte de las comunidades y la administración municipal. Y tercero, tiene que ver con las consecuencias de asumir un enfoque restringido para comprender y actuar frente al problema de seguridad. Finalmente, a partir de los hallazgos realizados, se presentan unas recomendaciones generales que permitan intervenir de manera más acertada los problemas de inseguridad en la ciudad.

\section{¿Desde dónde nos ubicamos?}

El enfoque de la seguridad humana desde abajo es la propuesta interpretativa bajo la cual se orientó la investigación. Este enfoque destaca las distintas interpretaciones, vivencias 
subjetivas, y percepciones que tienen las comunidades en la ciudad de Medellín ${ }^{1}$ frente al problema público de la seguridad en la ciudad y acerca de las políticas que el gobierno local implementa en esta materia.

Desde la apuesta de la investigación:

Nos colocamos deliberadamente del lado de los sectores más vulnerables y vulnerados de la ciudad, aquellos que experimentan la inseguridad de manera cotidiana y que en momentos de crisis son objeto también de las respuestas coercitivas que el Estado implementa para responder a las continuas demandas por reducir los índices más visibles de la inseguridad (OSHM, 2010:12)

A partir de este enfoque se pretende lograr una comprensión mejor de la política de seguridad en Medellín, no solamente desde la mirada oficial que brinda las autoridades gubernamentales de la ciudad, sino también desde la interpretación de los líderes y lideresas. Al analizar ambas aristas se pretende reconocer las distintas interpretaciones sobre la inseguridad en Medellín y se logra evidenciar para qué tipo de necesidades, amenazas o problemas se están creando estrategias de seguridad en la ciudad.

\section{Del discurso a la práctica, tránsito del enfoque humano al enfoque restringido}

En la época posterior a la Guerra Fría, se evidenció en la comunidad internacional un giro en la mirada en el tema de seguridad, en el cual los Estados ya no eran el centro en este tema; ¿Seguridad para quién? Es la pregunta fundamental que hay detrás de este giro, con la propuesta de seguridad humana, como su nombre lo presupone se trata de pensar precisamente en la seguridad para las personas, la cual es una de las tareas esenciales del Estado. En el caso de la ciudad de Medellín, específicamente en la administración municipal 2012-2015, se asume un enfoque de desarrollo humano integral y con este se compromete con un enfoque de seguridad amplio e integral que de acuerdo con el nombre de su plan de desarrollo y lema "Medellín un hogar para la vida", estaría centrado en las personas, en proteger la vida de quienes habitan la ciudad.

1 Se indaga esta percepción con líderes y lideresas comunitarias de la ciudad de Medellín, específicamente de la comuna 8 y 13, durante el periodo 2012-2013. 
El plan de desarrollo "Medellín un Hogar para la Vida", se construyó bajo dos principios fundamentales que tienen que ver con la Vida y la Equidad, sosteniendo la transversalidad de ambos componentes en relación con todo el plan. La apuesta de la administración municipal con el tema de la vida, está direccionada a "prevenir afectaciones a la población mediante el control de situaciones que atentan contra la vida y la seguridad humana" (Alcaldía de Medellín, 2012: 60).

En este sentido, si se consideran los planes de desarrollo² como el marco general y jerárquico de las políticas públicas, se podría afirmar que los temas incluidos en la construcción de los planes de desarrollo son posteriormente traducidos en políticas públicas (Cuervo, 2007), -por lo menos la gran mayoría de estos--, y por lo tanto como consecuencia de la inclusión de la necesidad de prevenir afectaciones a la seguridad humana en el plan de desarrollo "Medellín, un hogar para la vida", se podría esperar que las lecturas, decisiones y acciones de la actual administración en lo referente a política pública de seguridad, estén guiadas por un enfoque de seguridad humana o si no se desea ser tan explícitos, por lo menos por un enfoque de seguridad integral en consecuencia con lo planteado por el objetivo de la línea 1 del plan de desarrollo que es guía en materia de seguridad y se denomina: Ciudad que respeta, valora y protege la vida:

Garantizar a la población los derechos humanos fundamentales de seguridad y convivencia, salud, cultura, recreación y deporte, soportados en la familia como formador primario del ser humano, y con la participación y corresponsabilidad intersectorial, para que Medellín sea una ciudad segura que respeta, protege y disfruta la vida como valor supremo (Alcaldía de Medellin, 2012: 63)

Este objetivo y los principios de vida y equidad mencionados anteriormente, permitirían afirmar que las políticas públicas desarrolladas por la administración de Aníbal Gaviria, especialmente las referidas al tema de seguridad están relacionadas con un enfoque de seguridad humana; sin embargo, si se analizan las actuaciones de la administración municipal y las características esenciales de la seguridad humana: ser una preocupación

2 Con la ley 152 de 1994 se establece la Ley Orgánica del Plan de Desarrollo. 
universal $^{3}$, tener componentes interdependientes ${ }^{4}$, propender por la prevención más que por la reacción posterior y centrarse en el ser humano (Magaña Hernández, 2009:132), se encontrará la adopción de un enfoque restringido y tradicional de la seguridad, evidenciado en los siguientes aspectos:

1. Las problemáticas priorizadas en el Plan Integral de Seguridad y Convivencia - PISC $-{ }^{5}$ y las Estrategias ${ }^{6}$ adoptadas en dicho plan se centraron en problemáticas relacionadas con la criminalidad y las violencias como la interpersonal o intrafamiliar, atendiendo sólo las amenazas o afectaciones a la seguridad personal, dejando a un lado las demás afectaciones a las personas que surgen en la ciudad y que vulneran e impiden el pleno disfrute de sus derechos humanos. Durante el periodo analizado en el presente proyecto de investigación (2012-2013), una de las medidas del gobierno local más notorias y transmitidas es la inversión que se ha hecho en dotación a la fuerza pública para el cumplimiento de su labor legal y constitucional con una mayor efectividad. Desde el incremento en pie de fuerza, hasta la compra de vehículos dotados de tecnología ${ }^{7}$ la administración

3 Se entiende como preocupación universal la conciencia de "hay muchas amenazas que son comunes a todas las personas sin importar el nivel de desarrollo de sus países; tal es el caso del desempleo, el crimen, la contaminación, la violación de los derechos humanos, etcétera”. (Magaña Hernández, 2009:132).

4 Zagaña Hernández (2009), asume esta interdependencia como el que las afectaciones a la seguridad humana en un país tienen efectos en otros países, lo cual es muy viable. No obstante, hay otras interpretaciones a la característica de interdependencia y tienen que ver, con que la afectación a una de las dimensiones de la seguridad humana (económica, política, comunitaria, ambiental, en salud, personal y económica) afectada a su vez, varias o todas las demás dimensiones.

5 Afianzamiento y Sostenibilidad en el Tiempo de las Estructuras Criminales, Persistencia en la Ciudad de la Violencia Criminal; Rentas Criminales (Extorsión y Narcotráfico; Homicidio; Continuo Uso de Niños, Niñas, Adolescentes y Jóvenes por las Estructuras Criminales para la Comisión de Delitos; Desplazamiento Forzado; Hurtos; Violencia Interpersonal y Cotidiana; Violencia Intrafamiliar; Pérdida de Legitimidad de las Instituciones del Estado y de la Fuerza Pública.

6 Estrategia para el desmantelamiento y reducción de estructuras criminales y violencia criminal; Estrategia para la prevención del desplazamiento forzado, y el continuo uso de niños, niñas, adolescentes y jóvenes por parte de las estructuras criminales para la comisión de delitos; Estrategia para la atención y prevención de la violencia espontánea; Estrategia para la reducción del hurto a personas y el hurto de automotores; Estrategia de comunicación pública para el mejoramiento de la percepción de seguridad y divulgación de la gestión de las instituciones dentro del PISC.

7 Un ejemplo de ello es el aumento de 2000 policías entre 2013 y 2014, la meta de pasar de 170 a 500 cuadrantes, según declaración del, entonces, vicealcalde de Gobernabilidad, Seguridad y Servicio a la Ciudadanía, Luis 
municipal le ha apostado al fortalecimiento de la fuerza pública para combatir las estructuras criminales. En los territorios más vulnerables a la violencia criminal los incrementos han sido notables, incluso hasta llegar al punto de la densidad policiva excesiva, creando ambientes tensos, coercitivos y estigmatizantes.

2. El último argumento que ejemplifica la aplicación de un enfoque restringido en la administración municipal, se relaciona con los actores responsables de garantizar la seguridad en la ciudad, función que está en cabeza de la Secretaría de Seguridad, creada mediante Decreto 0495 de 2012, en el marco de la reestructuración municipal.

La Secretaría de Seguridad trabaja en coordinación con otras dependencias del municipio de Medellín y entidades del orden nacional como el Ejército Nacional, la Fiscalía General de la Nación, el Instituto Nacional de Medicina Legal y Ciencias Forenses, la Rama Judicial, el Ministerio Público, entre otros. Y cabe resaltar que en la formulación de la política pública de seguridad y convivencia participan actores de diversos sectores (educativos, económicos, sociales, fuerza pública, académicos, etc.) lo cual posibilitaría un enfoque integral; sin embargo, al hacer seguimiento a medios de comunicación impresos y consultar a líderes comunitarios en torno a las estrategias de seguridad implementadas hasta el día de hoy por la actual administración municipal, se encuentra una tendencia muy marcada a que la mayoría de estas acciones están en manos de organismos de justicia y policiales, lo cual desarrolla un enfoque de seguridad restringido y que no atiende a todas las inseguridades de los ciudadanos.

A manera de síntesis acerca del enfoque de seguridad de la Alcaldía de Medellín (2012-2013), los anteriores tres aspectos, presentan una idea general de por qué se afirma que hubo un tránsito entre el discurso y la práctica en materia de seguridad en la ciudad, especialmente porque no se están aplicando medidas centradas en el conjunto de amenazas a todos sus habitantes, si no solamente en las problemáticas relacionadas con la criminalidad, la violencia y afectaciones la vida, pero descuidando aspectos como el ambiental, el alimentario, el económico, la salud, el político y/o el comunitario.

Fernando Suárez: "manifestó que hoy existen 170 cuadrantes pero que la meta es llegar a los 500 el próximo año. Agrego que para diciembre se espera la llegada de 1.500 policías y 500 en el mes de marzo" (S.A. "Aumentaron cuadrantes". En: El Mundo, 28 de agosto de 2013). 
Es decir, que la administración municipal sólo se está centrando en ciertas amenazas a la seguridad, lo cual, según lo explica claramente Pérez de Armiño, se convierte en una manera de vaciar de contenido y aplicación práctica el concepto de seguridad humana:

\author{
limitarse a la "libertad frente al temor", es decir, a la protección frente a \\ la violencia física, olvidando la "libertad frente a la necesidad", o sea, la \\ seguridad que proporciona el desarrollo humano, supone revertir una de \\ las lineas de avance teórico que desembocaron en la gestación del concepto \\ de seguridad humana. (Pérez de Armiño, 2007: 74)
}

Vaciar de contenido el concepto de seguridad humana y adoptar un enfoque restringido de la seguridad, son asuntos que cuestiona esta investigación, ya que como se verá a continuación genera como consecuencias que existan divergencias entre las concepciones sobre la seguridad de la administración municipal y de las comunidades. Principalmente en relación a las causas de los fenómenos violentos que generan inseguridad en los territorios de la ciudad, según la concepción de las comunidades son fenómenos multicausales, teniendo dentro de sus orígenes, la pobreza, la exclusión y la falta de oportunidades; mientras que por parte de la administración municipal son factores generadores de inseguridad principalmente el narcotráfico y los actores armados; así mismo, las estrategias implementadas para solucionarlos deben estar dirigidas a varios aspectos de la vida social y no sólo ser de tipo militar, es decir, se propende por políticas integrales.

\title{
Divergencias y convergencias en cuanto a la construcción del problema de la seguridad en la ciudad de Medellín por parte de las comunidades y la administración municipal
} En este apartado se pretende identificar cuál ha sido la definición del problema de inseguridad en la política pública de seguridad en Medellín:

\footnotetext{
Los problemas de políticas públicas no son datos a priori, sino resultados de definiciones. Si una situación específica o un conjunto de circunstancias constituyen un problema y, por tanto, son un asunto capaz de despertar la preocupación del público, depende no sólo de los hechos sino también de las creencias y los valores. Pocas veces los hechos son claros y, aunque lo fueran, siempre están sujetos a diferentes interpretaciones (Cobb y Elder, 1996: 77).
} 
El problema de inseguridad en la ciudad de Medellín puede ser definido a través de distintos actores, que desde su concepción y valores pueden interpretar y definir el problema de maneras distintas. Esclarecer cuáles son estas definiciones del problema permite comprender las causas, las representaciones, las consecuencias, los afectados del problema y quiénes son los responsables o causantes de que exista dicho problema. Tener estas claridades es fundamental porque a partir de la definición aceptada, se empiezan a configurar las soluciones al problema. En esta medida, entender las divergencias y convergencias entre las definiciones de los actores institucionales y las comunidades permitirá identificar el grado de consenso que hay en la ciudad sobre el problema de in-seguridad.

El diagnóstico realizado por la administración de Aníbal Gaviria en relación con el tema de la seguridad arroja varias problemáticas principales, la primera problemática es la que más fuerza toma en relación a la ejecución de estrategias de seguridad, tiene que ver con la falta de control efectivo del territorio por parte de las autoridades policiales y judiciales y que se expresa en la:

Existencia de diversas modalidades delictivas, que se disputan el territorio buscando el control de rentas tanto legales como ilegales, producto de la extorsión y distintas modalidades de tráfico de drogas y armas, sumado a otras actividades y fenómenos (delitos contra la propiedad, secuestro, extorsión y homicidios), que vulneran el bienestar físico, psicológico y ambiental de la población y la vida en sociedad (Alcaldía de Medellín, 2012: 64).

Por lo discutido hasta acá, el hecho de reducir el problema de inseguridad a la falta de control efectivo del territorio por parte de autoridades policiales o judiciales conduce a propuestas de aumento de pie de fuerza; salidas que han sido protagonistas en la ciudad, no tanto por ser acciones exitosas, sino por insuficientes, en la medida en que muchos casos ha enfrentado a los habitantes a una inseguridad más, a partir de la vulneración de derechos humanos por parte de la fuerza pública. Por esto se presenta a continuación las distintas definiciones del problema de inseguridad desde la visión de las comunidades, esto permite en varios casos establecer un paralelo -en cuanto a divergencias y convergencias- con respecto a la mirada de la administración municipal.

Por su parte, las comunidades entienden el problema de inseguridad desde un con- 
junto de amenazas que no se ciernen solo sobre la seguridad personal, sino que atraviesa distintas afectaciones a dimensiones de la seguridad humana y que en conjunto revelan la lectura del problema de inseguridad -en las comunas 8 (Villa Hermosa) y 13 (San Javier)-: tiene que ver con las amenazas a las personas en el contexto de la planeación municipal, la amenaza ambiental, la falta de empleo y la estigmatización que sufren los habitantes de estos territorios. En relación con la amenaza a la seguridad que genera la planeación de la ciudad en los habitantes de la comuna 8 :

Uno se sorprende de ver cómo esta misma comunidad ve que le genera inseguridad la planeación desde la administración no teniendo en cuenta a los habitantes. Es que yo pienso que el mayor índice de inseguridad es la planeación desde las instituciones (Lideresa de la comuna 8, Medellín, 2013).

En la comuna 8 existen afectaciones concretas en relación a los megaproyectos que la administración municipal ha pensado para la comuna y que en muchos casos no son discutidos y priorizados por los habitantes de estos espacios. Una de las quejas que expresan es que en muchas ocasiones la implementación de estas estrategias obliga a expulsar personas de sus viviendas, tal como lo denuncia el informe del Observatorio de Seguridad Humana, en el marco de la estrategia de Cinturón Verde Metropolitano se han dado desalojos habitacionales en el barrio Villatina. ${ }^{8}$ Adicional a eso, el tema de la infraestructura como garantía de condiciones de seguridad es necesario mirarlo en detalle porque la apropiación de estos espacios por parte de la ciudadanía, obliga a concebir una planeación conjunta para conseguir aceptación y aprovechamiento de estos espacios en su ejecución.

En este mismo sentido, es importante mencionar que desde hace varios periodos las administraciones municipales han priorizado la dotación de infraestructuras en sectores con dificultades de seguridad en la ciudad, la cual, si bien es importante y necesaria, no es suficiente para lograr transformaciones reales y de fondo, así lo ven algunos habitantes de la Comuna 13, la cual es considerada como uno de los territorios de la ciudad más intervenidos, no sólo militarmente, sino también en infraestructura social:

8 Observatorio De Seguridad Humana De Medellín (2012). Informe situación de derechos humanos comuna 8. Fecha de consulta: 17 de septiembre de 2014, disponible en: http://goo.gl/6RFMe3 
la administración cree que haciendo más obras, más escaleras eléctricas, más metro-cables, más parque biblioteca la ciudad va a cambiar y no; porque ellos hacen las estructuras más no acompañan esas estructuras de un proceso social, transformador, sino que simplemente hacen la estructura y la dejan ahi y el problema de la estructura está, pero el problema social alrededor de esa estructura continúa y se agudiza incluso en varios casos. (Lider de la Comuna 13, Medellin, 2013).

Otra situación que constituye el problema de inseguridad en la comuna 8 de Medellín tiene que ver con las estrategias de movilidad en la ciudad que perjudican la seguridad ambiental de los habitantes de esta comuna:

\begin{abstract}
La movilidad en Medellín es el peor enemigo que puede tener el derecho a un ambiente sano; el tema de meterle movilidad que tranvias, que cables, ;no!, el tema aqui no es poner a andar mucho carro, mucho tranvía, mucho metro. Es que la gente no se mueva mucho de su territorio, que produzca en su territorio, que no se mueva de su territorio, habilitar ese espacio para ellos y habilitar ese hábitat, eso no se da. Son ideas de las comunidades frente a la planeación de las cosas, pero eso no se escucha (Lider de la comuna 8, Medellín, 2013).
\end{abstract}

Es importante esta mención que hace el líder comunitario en tanto evidencia que el tema de seguridad en la comuna 8 pasa por pensar en el tema ambiental, en el hábitat y en una planeación acertada por parte de las comunidades y de la administración municipal de estos territorios. Adicionalmente deja entrever las diferencias entre la administración municipal y las comunidades, la primera le apuesta a la movilidad, mientras que los segundos sostienen que es a través de la mejora del hábitat que se logra desarrollo y seguridad en sus comunas.

Dentro de esta definición del problema de in-seguridad son importantes las afirmaciones que permiten concretar las causas del problema, éstas tienen que ver con las situaciones socioeconómicas de los habitantes de la comuna 8 que los lleva a involucrarse en actuaciones delictivas que generen recursos para el sostenimiento de sus familias. Este involucramiento, podría sostenerse que, es forzado en la medida en que no hay condiciones de vida digna que les permita desarrollar su vida sin involucrarse en el conflicto. 
Sí bien es cierto, las precarias condiciones socioeconómicas no son las únicas generadoras de violencia, es decir, esta no es una relación directa e inmodificable de causa efecto, un contexto donde exista pobreza e inequidad, sumado a lógicas delictuales y de presiones sociales por obtener dinero rápidamente, si se convierte en un gran atizador de la violencia e inseguridad.

Adicional a las condiciones de pobreza, exclusión e inequidad, hay otra problemática que está ligada a ésta y es el de la estigmatización sufrida por los habitantes de las Comunas 13 (San Javier) y 8 (Villa Hermosa) en especial por los jóvenes; en este punto coinciden funcionarios públicos y líderes comunitarios. Así lo expresa la promotora de gobierno local de una de estas comuna "la comuna 13 está estigmatizada a nivel nacional e internacional por la guerra, por las muertes, por la violencia, como también por la inequidad" (Sandra María Bedoya, Medellín, 2013) y en este mismo sentido un líder de la comuna 8 menciona: "hablar de la comuna y decir el nombre siquiera o el número, ya es materia de estigma para no ingresar a un trabajo decente, entonces se tienen que adaptar los jóvenes en medio de unas actividades informales que no les revierten un nivel de vida digna”. (Líder de la comuna 8, Medellín, 2013). Esta estigmatización que sufren los habitantes de esta comuna en distintos espacios, les impide no solo acceder a un empleo sino que afecta asuntos psicológicos, el tener que negar su identidad y lugar de origen afecta situaciones en la seguridad de estas personas, es una necesidad social y personal poder reconocer estos aspectos.

La estigmatización se da tanto al exterior como al interior de la Comuna, algunos de sus líderes consideran que al exterior hay una estigmatización cultural, un señalamiento a nivel local, nacional, e incluso internacional: "[los jóvenes cuentan] que solo con nombrar que son de la 13 se sienten excluidos, hay gente que hasta se cambia de lugar y no está cerquita de ellos, no quieren tener nada que ver con ellos" (Lideresa de la Comuna 13, Medellín, 2013). Al interior de la Comuna, dicha estigmatización se da especialmente hacia los jóvenes por parte de las autoridades policiales:

El solo hecho de que los pelaos que cantan hip hop, que visten con sus ropas anchas; el solo hecho de que los estén cogiendo para requisar simplemente por sospecha. Ahi están violando el derecho a un joven a la libre expresión, a cómo se viste él. Aquí hubo un tiempo en que la policía los veía y ahímismo 
los requisaba. Creo que ahi es donde más se veía eso: el solo hecho de que un pelao saliera con su pantalón ancho, ya tenía un policía detrás. (Lider de la Comuna 13, Medellín, 2013)

Al momento de dar cuenta de las interpretaciones sobre el problema de inseguridad en la ciudad, es necesario reconocer no solo los hechos problemáticos, sino también los afectados. En esta ponencia se presenta especialmente el caso de la población joven como afectada por esta situación. Desde la administración municipal se consideran poblaciones altamente vulnerables por la violencia en la ciudad, la tasa de homicidios en la población joven en el 2011 fue de 181,8 por cada 100.000 jóvenes "lo que implica que éste es el grupo poblacional donde la ciudad debe focalizar sus esfuerzos en materia de seguridad y convivencia" (Alcaldía de Medellín, 2012: 68).

La definición del problema de inseguridad desde la institucionalidad tiene un acento marcado en los actores causantes del problema de inseguridad y no tanto en los afectados por el problema, por tanto las medidas implementadas por la administración municipal para enfrentar esta inseguridad, concretamente con los jóvenes, van en dos vías (ambas para los causantes del problema) la primera es de tipo reaccionaria y coercitiva, porque está intentado eliminar al enemigo a través del ataque de estructuras criminales, y la segunda es preventiva o disuasiva: los esfuerzos de la administración se han concentrado en mejorar las condiciones de este grupo poblacional, a través de estrategias relacionadas con la atención educativa y psicológica y el apoyo económico a jóvenes que se consideran en alto riesgo de pertenecer a grupos armados ilegales o que ya han iniciado a vincularse a dichos grupos. Esto a través de programas como "Jóvenes por la vida". Estas estrategias son muy polémicas, en tanto se consideran un estímulo indirecto para los jóvenes que deciden ingresar a la ilegalidad y a su vez limita la oferta a otros jóvenes no inmersos en el conflicto.

El decir de ellos [los jóvenes] si la alcaldía para que a mí me den un estudio o me reconozca un trabajo tengo que estar metido en un combo, porque eso lo ha demostrado la misma gobernabilidad si vos estas metido en un combo entonces te vamos a apoyar con un estudio entonces casi que obligan a los jóvenes a que estén allá, entonces es la parte más débil los jóvenes (Lider de la Comuna 13, Medellín, 2013). 
Por todo lo dicho hasta acá, las organizaciones sociales apuestan a que el enfoque en el tema de juventud no sea solo para atender los jóvenes en el conflicto, ya que gran cantidad de jóvenes han quedado excluidos de estas políticas y programas que están enfocadas a los victimarios. En esta medida, sostienen que la administración debe trabajar con la juventud que no está inmersa en el conflicto apostándole a sus iniciativas de arte y de cultura. Todo esto permite observar el desencanto por cuenta de los jóvenes de un conflicto que viven en sus territorios y también de la atención y acción del Estado en las comunas enfocada solo en el conflicto y no en las acciones de reivindicación de la vida desde las apuestas de los jóvenes. En este sentido un líder comunitario sostiene:

\footnotetext{
Pregunto a la Administración si seguirá promoviendo una política de muerte o de vida y si la apuesta seguirá siendo la guerra que como jóvenes decimos 'no' porque estamos cansados de seguir poniendo los muertos, y decimos no a la vinculación del conflicto y sus actores llámese como se llame y le decimos 'si' la música, al teatro, el cine el baile y la ciencia (Mesa de Articulación Juventud, Juan Camilo Hincapié Arboleda. 12/05/2012. Concejo de Medellin, acta 070: 144-145).
}

En este sentido, las medidas para contrarrestar el problema de inseguridad por parte de los actores gubernamentales en el municipio de Medellín, tiene un acento marcado en los actores causantes del problema de inseguridad y no tanto en los afectados por el problema. Se relega a los afectados por el problema, en términos de no reconocerlos como víctimas o de no realizar acciones contundentes que desde la prevención mejoren sus condiciones como grupos vulnerables ante el conflicto que vive la ciudad.

En síntesis, los problemas de los que parten las políticas públicas de seguridad en la ciudad de Medellín, desconocen una realidad evidenciada por los sectores que sufren a diario la situación de inseguridad. Existen distintos tipos de afectaciones a la seguridad humana de los habitantes, y que para ellos es reconocido como inseguridad en Medellín, es decir resulta ser el problema que resaltan las comunidades y que al parecer no es el que buscan atacar las políticas públicas de seguridad en la ciudad de Medellín.

Por tanto, no partir de una definición común, entre los afectados por el problema de inseguridad y los tomadores de decisiones conlleva a que en la implementación de deci- 
siones se re victimice a los afectados por el problema de inseguridad en Medellín y en esa medida, que las estrategias no estén enfocadas en la lectura del problema que tienen las comunidades, quienes en últimas son quienes viven las afectaciones diariamente.

¿Cuáles son las implicaciones de la implementación de un enfoque de seguridad restringido en Medellín?

Los efectos de la adopción y aplicación de políticas públicas de seguridad de corte restrictivo o militarista en Medellín, han tenido graves consecuencias tanto para la institucionalidad como para las comunidades de la ciudad, que son quienes, como se ha insinuado, en sus vidas cotidianas padecen las situaciones y hechos de inseguridad. Sin embargo, en la investigación se encontraron principalmente dos efectos, por un lado el que se acentúe la falta de legitimidad de las instituciones públicas y por otro lado la vulneración a los derechos humanos, lo cual a su vez se convierte en obstáculo para la generación de condiciones de vida seguras.

Respecto a la acentuación o generación de deslegitimidad de las instituciones de la alcaldía municipal y de la fuerza pública en la ciudad, es pertinente precisar que es gracias a la adopción de un enfoque de seguridad humana que se ha logrado tener claridades al respecto, pues tal como lo afirma Pérez de Armiño "Así pues, la seguridad humana actúa como un referente que coloca al Estado ante el escrutinio crítico, que permite juzgar sus políticas, sus capacidades, sus relaciones con los ciudadanos e incluso su legitimidad". (2007:68).

En este caso se ha podido, por una parte verificar la deslegitimidad que ya existía de las instituciones públicas, la cual incluso había sido priorizada como una de las problemáticas que más afectan la seguridad y la convivencia en la ciudad, en el PISC, nombrándola como: "Pérdida de legitimidad de las instituciones del Estado y de la fuerza pública"; y por otro lado se ha visto como ésta, ya débil legitimidad, se reduce más, debido a la falta de efectividad de las políticas públicas de seguridad y al no recoger las necesidades, problemáticas y propuestas de las comunidades, lo cual ocurre por la falta de reales condiciones de participación para los ciudadanos. Sumado a esto, una razón fundamental para la pérdida de legitimidad de la institucionalidad, está directamente relacionada con los casos de corrupción y violación a los derechos humanos por parte de la fuerza pública, el cual es el tema a tratar a continuación como efecto de la adopción de enfoques militaristas. 
La relación entre las políticas públicas de seguridad adoptadas por parte de la administración municipal de Medellín y los derechos humanos, se da en dos sentidos, el primero tiene que ver con acciones directas cometidas por policías o militares que violan los derechos humanos y el segundo con que la adopción de ciertas estrategias de seguridad o en general estrategias de la administración municipal, conducen a la vulneración de ciertos derechos.

Respecto a las acciones directas, los casos más nombrados son los cometidos por la fuerza pública, hay quejas hacia el ejército relacionadas en su mayoría con las batidas que realiza y que impiden el ejercicio de la objeción de conciencia, pero especialmente hay otro ejemplos, en los cuales la policía es quien tiene el mayor número de quejas en la ciudad:

La concentración de quejas por vulneraciones a los DD.HH. se registra en cabeza de la Policía Nacional, quien realiza intervención en el territorio con grupos regulares de policías, reforzados con grupos especiales o de choque como Grupo de Operaciones Especiales -GOES-que intervienen en las comunas 8 (Villa hermosa) y 13 (San Javier); la Fuerza de Comando Urbano -FUCUR-, en la Comuna 13 (San Javier), y el Escuadrón Móvil Antidisturbios -ESMADque actúa en el control de masas y restablecimiento del orden público. (Personería de Medellín, 2013: 40-41).

Uno de los hechos que posibilitan vulneraciones a los derechos humanos es la connivencia de la fuerza pública con grupos armados ilegales, pero también hay acciones directas que van desde las mencionadas batidas realizadas por el ejército que irrespetan el ejercicio del derecho a ser objetores de conciencia por parte de los jóvenes, la realización de allanamientos ilegales o detenciones arbitrarias que afectan el derecho al debido proceso, las restricciones a la circulación por los diferentes territorios que impiden la movilidad (derecho a la locomoción), hasta casos de homicidios y torturas por parte de la policía con evidentes afectaciones al derecho a la vida e integridad física ${ }^{9}$ :

9 Igualmente se ha hecho referencia por parte de líderes comunitarios, de afectaciones a la integridad psicológica, no sólo por el miedo que se genera en los ciudadanos, sino también por casos como los denunciados en la Comuna 13 dónde han atemorizado a los jóvenes responsabilizándolos de delitos que recién hayan ocurrido en la Comuna o simplemente porque son estigmatizados por su forma de vestir: "el solo hecho de que los pelaos que cantan hip hop, que 


\section{Grafico 1}

Número de Quejas recibidas por presuntas vulneraciones a los DDHH y al DIH por miembros de la fuerza pública, según mes para el periodo 2012-2013
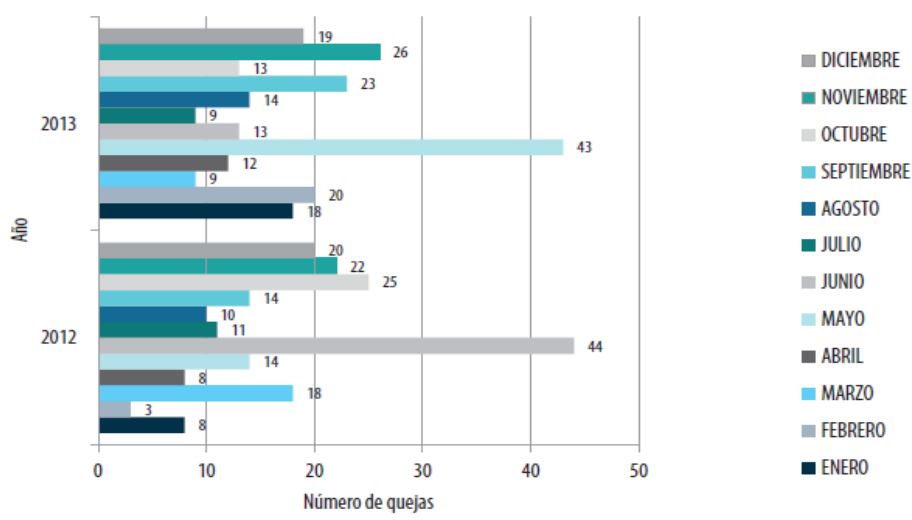

Fuente: Personería de Medellin (2014). Informe sobre la situación de los derechos humanos en la ciudad de Medellin (2013:84).

Entre los años 2012 y 2013, la Personería de Medellín recibió 413 quejas por la actuación de miembros de la fuerza pública, en 2012 "7 fueron infracciones al DIH y 190 vulneraciones a los DD.HH." ${ }^{10}$ (Personería de Medellín, 2013); mientras que en 2013 se registraron 216 quejas. ${ }^{11}$

Finalmente, para precisar las acciones de seguridad, que pueden terminar generando mayor inseguridad debido a la vulneración de los derechos humanos o a las limitaciones en sus garantías, a continuación se analizará un caso emblemático que brinda mayor claridad al respecto.

ellos visten con sus ropas anchas; el solo hecho de que los estén cogiendo para requisar simplemente por sospecha. Ahí están violando el derecho a un joven a la libre expresión, a cómo se viste él”. (Líder de la Comuna 13, Medellín, 2013)

10 De acuerdo con reportes de la Policía MEVAL, durante el 2012 se recibieron 1.273 quejas que dieron como resultado según investigación realizada por la Oficina de Control Interno de la propia MEVAL que siete quejas fueron sancionadas y 85 archivadas (Personería de Medellín, 2013: 41).

11 "Con relación a este tema la Personería de Medellín registró 216 quejas por presuntas vulneraciones a los derechos humanos cometidas por miembros de la fuerza pública, esto para el período comprendido entre enero y diciembre de 2013. De estas, 176 fueron contra la Policía Nacional, 26 contra el Ejército Nacional y 14 contra el Escuadrón Móvil Anti-Disturbios (ESMAD)". (Personería de Medellín, 2014: p 81) 


\section{Programa Jóvenes por la vida}

Es una estrategia de la administración municipal relacionada con proyectos y programas dirigidos a jóvenes mediante oferta económica, educativa, cultural, deportiva y de empleo. Pese a que en el plan de desarrollo "Medellín un hogar para la vida" se contemplan seis componentes del programa Jóvenes por la vida ${ }^{12}$, de los cuales sólo dos están dirigidos o tienen un foco en jóvenes en riesgo de pertenecer a grupos armados ilegales o que ya pertenecen a éstos, en el imaginario de las comunidades persiste la idea de que ésta es una estrategia que termina generando una sensación de desigualdad, puesto que los demás jóvenes que no se encuentran en situación de riesgo (ingresar a la ilegalidad) también tienen necesidad de que sus derechos sean garantizados.

Lo que evidencian las comunidades es que se les están dando estímulos principalmente económicos a los jóvenes que están en grupos armados ilegales, llámense combos o bandas: "En el territorio uno no ve políticas públicas pagándole a los muchachos 350 mil pesos, a los pelaos, a los de los combos para que hagan cuestiones culturales" (Líder de la Comuna 8, Medellín, 2013). Lectura de las comunidades que expresa críticas desde diversos sectores cuando a la mayoría de jóvenes no se les garantiza el acceso a la educación superior, el primer empleo o la oferta de actividades culturales y deportivas.

La apreciación en esta investigación, es que si bien es cierto, deben existir políticas públicas para tratar la situación de los jóvenes vinculados al conflicto, se debe partir de una concepción general y es que los jóvenes, al igual que la población general tienen unos derechos humanos que les deben ser garantizados, y no sólo pensarse en políticas de oferta de oportunidades a los jóvenes como una forma de que no se vinculen a lo ilegal, porque esto en principio tendría dos problemas, el primero es que restringiría las ofertas institucionales sólo a los jóvenes que viven en contextos en los cuales son susceptibles de vincularse a acciones o grupos ilegales, dejando por fuera a quienes no lo están; y el segundo es que las agendas de seguridad de la administración

12 Jóvenes por la vida, una estrategia de convivencia; Jóvenes por la vida - Medellín sana y libre de adicciones; Jóvenes por la vida, una estrategia cultural; Jóvenes por la vida, una estrategia deportiva y recreativa; Jóvenes por la vida, una estrategia de oportunidades; Jóvenes por la vida, una estrategia para la habilitación e inserción laboral. (Alcaldía de Medellín, 2012a: 336-345) 
municipal a las cuales se estaría atendiendo son las de los grupos armados, dejando de lado las propuestas por el resto de la población.

Adicional a esto, otro argumento para exigir a la administración municipal ofertas institucionales que contribuyan a la garantía, protección y defensa de los derechos humanos de todos los jóvenes, es que los derechos humanos, a diferencia del derecho internacional humanitario, son de carácter permanente y por tanto se deben garantizar en todo tiempo y lugar.

\section{Reflexiones finales}

Para trascender la visión restringida de las políticas públicas de seguridad, el foco de dichas políticas debe estar en las causas de las inseguridades y no en los efectos o consecuencias, puesto que el centrarse en los efectos lleva a que en diversas instituciones y niveles se implementen políticas de resultados, pero sin lograr transformaciones profundas y preventivas. No obstante, es importante precisar que al momento de revisar las causas, se requiere tener en cuenta que las problemáticas de seguridad son multicausales, en ese sentido, las políticas públicas de seguridad y convivencia deben ser integrales y atendiendo a las múltiples causas de las inseguridades.

Por otra parte y para finalizar, un llamado de atención urgente para el alcalde de la ciudad y el vicealcalde de gobernabilidad, seguridad y servicio a la ciudadanía y todo su equipo de trabajo, tiene que ver con la necesidad de poner atención a la planeación de la ciudad, por nuestros estudios de caso (Comunas 8 y 13), la inseguridad a la que se alude en el tema de planeación está relacionada principalmente con vulneraciones al derecho a la vivienda y el derecho a la alimentación; no obstante, al escuchar a líderes de otros territorios de la ciudad y hacer un seguimiento a medios de comunicación se evidencia que la inseguridad asociada a la planeación está relacionada también con afectaciones al ambiente, problemas de infraestructura en construcciones públicas y privadas y a la generación de focos de inseguridad tras la realización de reubicaciones. Es por ello que se solicita a los funcionarios responsables de atender el problema de inseguridad a la ciudad que se articulen con los funcionarios de planeación y realicen alertas y acciones que prevengan futuras y graves inseguridades. 


\section{Bibliografía}

ALCALDÍA DE MEDELLÍN (2012a.) Plan de Desarrollo 2012- 2015: Medellín, un Hogar para la Vida, Medellín. Disponible en: http://www.medellin.gov.co/irj/portal/ciudadanos? NavigationTarget=navurl://90b954e4cc54924b972c3ac7e05be7dc

ALCALDÍA DE MEDELLÍN (2012b.). Plan Integral de Seguridad y Convivencia 2012-2015. http://www.medellin.gov.co/irj/portal/ciudadanos?NavigationTarget=navur 1://81f0b832072cf638c5f853401a3e1070

ALCALDÍA DE MEDELLÍN (2012c.) Decreto 0495 del 2012 http://www.medellin. gov.co/irj/go/km/docs/wpccontent/Sites/Subportal\%20del\%20Ciudadano/Convivencia\%20y\%20seguridad/Secciones/Normas/Documentos/2012/Decreto\%200495.pdf

COBB, W. y Elder, D. (1996). Formación de la agenda. El caso de la política de los ancianos. En L. F. Aguilar Villanueva, problemas públicos y agenda de gobierno (págs. 18977104). México: Miguel Ángel Porrúa.

OSHM - Observatorio de Seguridad Humana de Medellín, (2012) control territorial y resistencias: una lectura desde la seguridad humana, Medellín, La carreta editores E.U., pp. 81-115.

PEREZ DE ARMIÑO, K. (2007). El concepto y el uso de la seguridad humana: análisis crítico de sus potencialidades y riesgos. En: Seguridad Humana: conceptos, experiencias y propuestas. Revista CIDOB D'AFERS INTERNACIONALS (76). Fundación CIDOB: Barcelona, Diciembre-enero.

PERSONERÍA DE MEDELLÍN (2013). Informe sobre la situación de los derechos humanos en la ciudad de Medellín 2012.

PERSONERÍA DE MEDELLÍN (2014). Informe sobre la situación de los derechos humanos en la ciudad de Medellín 2013.

PINZÓN, E. M. R. (2009). "Las narrativas de los conflictos y la construcción de un enfoque de la seguridad humana. Análisis crítico del caso de Colombia”. En: Revista CS, núm. 3, 125-146. Cali: Universidad Icesi 
MAGAÑA HERNÁNDEZ, D. M. (2009). El otro paradigma de la seguridad. En: Alegatos (72). México, mayo-agosto, (Pp. 127-150).

MEJÍA MOLINA, D. C. (2009). "Efectos para Colombia de los debates en torno a la seguridad del Estado y a la seguridad humana”. En: Revista CS, núm. 3, 109-123. Cali: Universidad Icesi

Fuentes normativas

Concejo de Medellín. (12/05/2012) acta 070. Disponible en: http://www.concejodemedellin.gov.co/ Fecha de consulta: 13 de julio de 2013.

\section{Entrevistas}

Entrevista a lideresa comuna 8, noviembre 5 de 2013.

Entrevista grupal a líderes Comuna 13, octubre 23 de 2013.

Entrevista a lideresa Comuna 13, octubre 29 de 2013.

Entrevista a líder comuna 8, octubre 15 de 2013.

Entrevista a Sandra María Bedoya. Promotora Local de Gobierno Comuna 13. Secretaría de Gobierno y Derechos Humanos, octubre 28 de 2013. 